\title{
A putative causal relationship between genetically determined female body shape and posttraumatic stress disorder
}

\section{Citation}

Polimanti, R., A. B. Amstadter, M. B. Stein, L. M. Almli, D. G. Baker, L. J. Bierut, B. Bradley, et al. 2017. "A putative causal relationship between genetically determined female body shape and posttraumatic stress disorder." Genome Medicine 9 (1): 99. doi:10.1186/s13073-017-0491-4. http://dx.doi.org/10.1186/s13073-017-0491-4.

\section{Published Version}

doi:10.1186/s13073-017-0491-4

\section{Permanent link}

http://nrs.harvard.edu/urn-3:HUL.InstRepos:34651976

\section{Terms of Use}

This article was downloaded from Harvard University's DASH repository, and is made available under the terms and conditions applicable to Other Posted Material, as set forth at http:// nrs.harvard.edu/urn-3:HUL.InstRepos:dash.current.terms-of-use\#LAA

\section{Share Your Story}

The Harvard community has made this article openly available.

Please share how this access benefits you. Submit a story.

Accessibility 


\title{
A putative causal relationship between genetically determined female body shape and posttraumatic stress disorder
}

Renato Polimanti ${ }^{1 *}$, Ananda B. Amstadter ${ }^{2}$, Murray B. Stein ${ }^{3,4,5}$, Lynn M. Almli6 ${ }^{6}$ Dewleen G. Baker ${ }^{3,5}$, Laura J. Bierut ${ }^{7}$, Bekh Bradley ${ }^{6,8}$, Lindsay A. Farrer ${ }^{9}$, Eric O. Johnson ${ }^{10}$, Anthony King ${ }^{11}$, Henry R. Kranzler ${ }^{12}$, Adam X. Maihofer ${ }^{3}$, John P. Rice ${ }^{7}$, Andrea L. Roberts ${ }^{13}$, Nancy L. Saccone ${ }^{14}$, Hongyu Zhao ${ }^{15}$, Israel Liberzon ${ }^{11,16}$, Kerry J. Ressler ${ }^{17,18}$, Caroline M. Nievergelt ${ }^{3,5}$, Karestan C. Koenen ${ }^{19,20,21}$, and Joel Gelernter ${ }^{1,22}$ for The Psychiatric Genomics

Consortium Posttraumatic Stress Disorder Workgroup

\begin{abstract}
Background: The nature and underlying mechanisms of the observed increased vulnerability to posttraumatic stress disorder (PTSD) in women are unclear.

Methods: We investigated the genetic overlap of PTSD with anthropometric traits and reproductive behaviors and functions in women. The analysis was conducted using female-specific summary statistics from large genome-wide association studies (GWAS) and a cohort of 3577 European American women (966 PTSD cases and 2611 traumaexposed controls). We applied a high-resolution polygenic score approach and Mendelian randomization analysis to investigate genetic correlations and causal relationships.

Results: We observed an inverse association of PTSD with genetically determined anthropometric traits related to body shape, independent of body mass index (BMI). The top association was related to BMl-adjusted waist circumference ( $W C_{\text {adj; }} R=-0.079, P<0.001, Q=0.011$ ). We estimated a relative decrease of $64.6 \%$ (95\% confidence interval $=27.5-82.7$ ) in the risk of PTSD per 1-SD increase in $W C_{\text {adj. }}$ MR-Egger regression intercept analysis showed no evidence of pleiotropic effects in this association $\left(P_{\text {pleiotropy }}=0.979\right.$ ). We also observed associations of genetically determined $W C_{\text {adj }}$ with age at first sexual intercourse and number of sexual partners ( $P=0.013$ and $P<0.001$, respectively).
\end{abstract}

Conclusions: There is a putative causal relationship between genetically determined female body shape and PTSD, which could be mediated by evolutionary mechanisms involved in human sexual behaviors.

Keywords: Trauma, Genetics, Women, Anthropometric traits

\section{Background}

Posttraumatic stress disorder (PTSD) is a mental illness that affects susceptible individuals who have experienced, witnessed, or been confronted with an event involving actual or threatened dangers [1]. Individuals affected by PTSD present symptoms that, according to the DSM-5, include four diagnostic clusters (re-

\footnotetext{
* Correspondence: renato.polimanti@yale.edu

'Department of Psychiatry, Yale University School of Medicine and VA CT Healthcare Center, 116A2, 950 Campbell Avenue, West Haven, CT 06516, USA Full list of author information is available at the end of the article
}

experiencing, avoidance, negative cognitions and mood, and arousal) [2]. At least one in nine American women meet lifetime diagnostic criteria for PTSD and women's risk of PTSD is twice that of men [3]. Among women, PTSD is also more likely to be chronic and associated with greater functional impairment and distinct neurobiological profiles $[4,5]$. Evidence suggests that women are more vulnerable than men to develop PTSD when exposed to similar traumas [6].

Women's higher risk for PTSD likely arises from biologic and social/environmental factors. For example, sex differences in trauma exposure may partially explain the differential risk for PTSD. Women are more likely to be 
exposed to sexual violence, which has a high conditional risk of PTSD [7]. Alternatively, the pattern of results from twin studies suggests that PTSD may be more heritable in women than in men $[8,9]$ and results from a recent genome-wide investigation also demonstrate higher molecular heritability in women [10]. Sex hormones, particularly estradiol and progesterone, have been implicated as mediators of this risk, suggesting that their effects on biological, behavioral, and cognitive pathways augment the vulnerability to PTSD [11]. A recent epigenome-wide association study supported this possible mechanism; it reported an estrogen-dependent association of HDAC4 with fear in female mice and women with PTSD [12].

Genetic data can be used to investigate this complex network of multiple factors. Polygenic risk score (PRS) analyses and Mendelian randomization (MR) studies are less biased by confounders than observational studies and can be used to investigate the predisposition to complex traits, identifying shared molecular pathways, pleiotropy, and causal relationships [13, 14]. Here, we used summary statistics from female-specific genomewide association studies (GWAS) of traits that may be related to disease pathogenesis-i.e. anthropometric characteristics [15], reproductive physiology and behaviors [16], and educational attainment [17] - to examine the mechanisms underlying women's vulnerability to PTSD. Although these traits were previously indicated as related to PTSD in women, testing a wide range of genetic correlations can provide useful etiological insights and help prioritize likely causal relationships [18]. A greater understanding of the biological and external social processes involved in PTSD will help to address the critically important interplay between two important public health issues: PTSD and women's health.

\section{Methods \\ Cohorts from the Psychiatric Genomics Consortium PTSD Workgroup}

The individual data from the subjects included were made available by the members of the Psychiatric Genomics Consortium PTSD Workgroup. Details regarding participants, genotyping, quality control, imputation, and ancestry assignment were reported previously [10]. In the present study, we used data regarding female participants from seven cohorts: Collaborative Genetic Study of Nicotine Dependence [19]; Family Study of Cocaine Dependence [20]; Yale-Penn [21]; Grady Trauma Project [22]; Marine Resilience Study [23]; Nurses' Health Study [24]; and Ohio National Guard [25]. Because the individuals in the GWAS that were used as sources for summary statistics for the traits of interest were of European descent, we restricted our analysis to women of that population. Quality control criteria for ancestry assignment and sex check are reported in our previous publication [10]. We considered imputed genotypes to maximize a consistent SNP (Single Nucleotide Polymorphism) panel between the training and testing sets. Imputed SNPs with high imputation quality (genotype call probability $\geq 0.8$ ), minor allele frequency $\geq 1 \%$, missingness per marker $\leq 5 \%$, missingness per individual $\leq 5 \%$, and Hardy-Weinberg equilibrium $P>10^{-4}$ were retained. After applying these quality control criteria, we retained information on 4,875,110 variants in a final sample of 3577 women (966 PTSD cases and 2611 trauma-exposed controls). To investigate the specificity of our findings for PTSD in women, we investigated a sample of 4628 men (501 PTSD cases and 4127 traumaexposed controls) from the same cohorts of the Psychiatric Genomics Consortium PTSD Workgroup. The same quality control criteria were applied to the data from men. Principal component analysis of the final sample was conducted using PLINK 1.9 [26] after linkage disequilibrium (LD) pruning $\left(\mathrm{R}^{2}<0.2\right)$.

\section{Polygenic risk score analysis}

We conducted cross-phenotype PRS analyses using PRSice software [27] (available at http://prsice.info/). This method permits testing whether the polygenic components of inheritance have a substantial effect on multiple complex traits [28]. For polygenic profile scoring, we used summary statistics generated from female-specific GWAS of 17 traits (Table 1) conducted by the Genetic Investigation of ANthropometric Traits (GIANT) Consortium [29-32], the Reproductive Genetics (Reprogen) Consortium [33, 34], and the Social Science Genetic Association Consortium (SSGAC) $[35,36]$. None of the PGC-PTSD cohorts was included in these previous GWAS and, thereby, no large overlap is expected among these samples. We considered multiple association $P$ value thresholds ( $\mathrm{PT}=10^{-8}, 10^{-7}, 10^{-6}, 10^{-5}, 10^{-4}, 0.001,0.05,0.3,0.5$ ) for SNP inclusion, i.e. we conducted a high-resolution analysis. The PRS were calculated after using $P$ valueinformed clumping with a LD cut-off of $\mathrm{R}^{2}=0.3$ within a 500-kb window, and excluding the Major Histocompatibility Complex region of the genome because of its complex LD structure. The PRS that were generated were fitted in regression models with adjustments for the top ten ancestry principal components to calculate Nagelkerke's $\mathrm{R}$ as the figure of merit for prediction ability. We applied false discovery rate (FDR) multiple testing correction $(\mathrm{Q}<0.05)$ to correct for the number of PRS and thresholds tested (a total of 170 tests). A gene ontology (GO) enrichment analysis was conducted based on the PRS results: the variants included in the significant PRS with direction concordant with PRS direction were considered in the enrichment analysis. Variants were mapped to the nearest genes and then the gene loci were entered 
Table 1 GWAS summary statistics used for polygenic risk scoring

\begin{tabular}{|c|c|c|c|c|c|}
\hline Consortium & Trait & Abbreviation & Women N & Variant N & Data link \\
\hline \multirow{12}{*}{$\begin{array}{l}\text { Genetic Investigation of } \\
\text { ANthropometric Traits (GIANT) }\end{array}$} & Body mass index & $\mathrm{BMl}$ & 128,698 & $2,494,584$ & \multirow{12}{*}{$\begin{array}{l}\text { http://portals.broadinstitute.org/collaboration/giant/ } \\
\text { index.php/GIANT_consortium_data_files }\end{array}$} \\
\hline & $\begin{array}{l}\text { BMI (age } \leq \\
50 \text { years) }\end{array}$ & $\mathrm{BMI}_{<50}$ & 52,270 & $2,407,668$ & \\
\hline & $\begin{array}{l}\text { BMl (age } \geq \\
50 \text { years) }\end{array}$ & $\mathrm{BMI}_{>50}$ & 86,731 & $2,407,629$ & \\
\hline & Height & Height & 64,811 & $2,748,519$ & \\
\hline & Hip circumference & HIP & 85,973 & $2,466,814$ & \\
\hline & $\begin{array}{l}\text { HIP adjusted for } \\
\text { BMI }\end{array}$ & $H I P_{a d j}$ & 84,787 & $2,466,814$ & \\
\hline & $\begin{array}{l}\text { Waist } \\
\text { circumference }\end{array}$ & WC & 90,543 & $2,444,344$ & \\
\hline & $\begin{array}{l}\text { WC adjusted for } \\
\text { BMI }\end{array}$ & $W C_{a d j}$ & 89,759 & $2,473,027$ & \\
\hline & Waist-hip ratio & WHR & 85,466 & $2,466,093$ & \\
\hline & $\begin{array}{l}\text { WHR adjusted for } \\
\text { BMI }\end{array}$ & WHR adj & 84,222 & $2,467,768$ & \\
\hline & $\begin{array}{l}\text { WHRadj (age } \leq \\
50 \text { years) }\end{array}$ & $W H R_{a d j<50}$ & 34,523 & $2,366,527$ & \\
\hline & $\begin{array}{l}\text { WHRadj (age } \geq \\
50 \text { years) }\end{array}$ & $W H R_{\text {adj }>50}$ & 56,150 & $2,366,567$ & \\
\hline \multirow{2}{*}{$\begin{array}{l}\text { Reproductive Genetics } \\
\text { (Reprogen) }\end{array}$} & Age at menarche & Menarche & 182,416 & $2,441,881$ & \multirow[t]{2}{*}{ http://www.reprogen.org/data_download.html } \\
\hline & $\begin{array}{l}\text { Age at } \\
\text { menopause }\end{array}$ & Menopause & 69,360 & $2,418,695$ & \\
\hline \multirow{3}{*}{$\begin{array}{l}\text { Social Science Genetic } \\
\text { Association Consortium (SSGAC) }\end{array}$} & Age at first birth & AFB & 158,856 & $2,470,136$ & \multirow[t]{3}{*}{ http://www.thessgac.org/data } \\
\hline & Education years & EDU & 122,427 & $7,643,300$ & \\
\hline & $\begin{array}{l}\text { Number of } \\
\text { children ever born }\end{array}$ & NEB & 222,837 & $2,471,862$ & \\
\hline
\end{tabular}

into the enrichment analysis conducted using the PANTHER v11.1 Overrepresentation Test (release 20160715; Reference List: Homo Sapiens) [37]. FDR correction was applied to the enrichment results to account for multiple testing. The GO enrichment results were further investigated using REVIGO [38]. Specifically, GO enrichments were used to make a graph-based visualization considering an allowed similarity of 0.7 , UniProt as reference database, and SimRel method as the semantic similarity measure.

\section{Two-sample Mendelian randomization analysis}

The two-sample MR analysis was conducted using the $\mathrm{R}$ package MendelianRandomization (available at https:// cran.r-project.org/web/packages/MendelianRandomization /index.html) and the GWAS summary statistics. This is a strategy to test cause-effect associations, in which evidence on the associations of genetic variants (i.e. instrumental variable) with the risk factor (i.e. exposure) and with the outcome comes from two sources [39]. We used SNPs associated with waist circumference adjusted for body mass index (BMI) $\left(\mathrm{WC}_{\text {adj }} \mathrm{P}<10^{-8} N=31\right.$ ), which showed the most significant correlation with PTSD in our
PRS analysis (FDR Q $=0.011$ ), as the instrumental variable. The SNP-exposure and SNP-outcome coefficients $\left(\mathrm{WC}_{\text {adj }}\right.$ and PTSD associations, respectively) were combined using an inverse-variance-weighted approach to give an overall estimate of the causal effect. To verify the stability of the results, we compared the findings obtained from inversevariance-weighted analysis to the results of other MR approaches, including simple median, weighted median, and MR-Egger regression. Different methods have sensitivities to different potential issues, accommodate different scenarios, and vary in their statistical efficiency. Comparing the results of different methods permits validation of the analyses. MR-Egger regression intercept was considered to test for the presence of pleiotropic effects of the SNPs on the outcome [40]. Due to the lack of genome-wide significant loci for PTSD, it was not possible to apply reverse $\mathrm{MR}$ to test the effect of PTSD-associated alleles on $\mathrm{WC}_{\mathrm{adj}}$ to confirm the direction of the causal relationship between these traits [41]. However, we used a reverse PRS analysis of PTSD to predict $\mathrm{WC}_{\text {adj }}$ and other anthropometric traits based on GWAS summary statistics. This reverse PRS analysis was conducted using PRSice software [27]. To 
follow up a possible "evolutionary link" between female body shape and PTSD, we tested the association of genetically determined $\mathrm{WC}_{\text {adj }}$ with age at first sexual intercourse (AFS) and number of sexual partners (NSP). For AFS, we used female-specific summary statistics from a recent GWAS, including women of European descent only [42]. This previous GWAS was conducted considering a range of AFS, which also includes a small fraction of individuals who had their first sexual intercourse before 12 years of age. For NSP, we used UK Biobank data on a total of 58,356 unrelated women of European descent. We calculated the association of the top 31 SNPs associated with $\mathrm{WC}_{\text {adj }}\left(P<10^{-8}\right)$ with NSP using a linear regression model. Before being entered into the analysis, NSP was adjusted for age and the top ten ancestry principal components and then normalized using appropriate Box-Cox power transformations.

\section{Data availability}

Data supporting the findings of this study are available within the article and its Additional files. GWAS Summary level data used to calculate PRS in this study were obtained from links reported in Table 1.

\section{Results}

We used summary statistics generated from femalespecific GWAS of 17 traits (Table 1) to yield polygenic profile scoring and tested the association of the derived PRS with PTSD considering multiple PT and using samples from the Psychiatric Genomics Consortium PTSD Workgroup (966 PTSD cases and 2611 trauma-exposed controls). Six PRS showed correlations with PTSD that survived FDR multiple testing correction $(\mathrm{Q}<0.05$; Table 2; Additional file 1). Although LD score regression is designed to analyze large sample sizes [18], using this method we nevertheless replicated the directions of five correlations and two of them survived multiple testing correction (Additional file 2). The strongest PRS result was observed for $\mathrm{WC}_{\mathrm{adj}}$ : women with high genetically predicted $\mathrm{WC}_{\text {adj }}$ have low PTSD risk (PT: $10^{-8} ; \mathrm{R}=-0.079, \mathrm{P}$ $<0.001, \mathrm{Q}=0.011)$. Additional file 3 shows the distribution of $\mathrm{WC}_{\mathrm{adj}}$ PRS in PTSD cases and controls. The same variants included in the $\mathrm{WC}_{\text {adj }}$ PRS also drove four of the other associations observed: waist-hip ratio (WHR, PT = $\left.10^{-7} ; \mathrm{R}=-0.064, P=0.001, \mathrm{Q}=0.023\right)$; WHR adjusted for BMI (WHR adj,$\quad \mathrm{PT}=10^{-7} ; \mathrm{R}=-0.059, \quad P=0.001, \quad \mathrm{Q}=$ $0.030)$, WHRadj in women aged $<50$ years $\left(W_{\text {adj }}<50\right.$, $\left.\mathrm{PT}=10^{-6} ; \quad \mathrm{R}=-0.076, \quad P=1.25^{*} 10^{-4}, \quad \mathrm{Q}=0.011\right), \quad$ and $\mathrm{WHR}_{\text {adj }}$ in women aged $>50$ years $\left(\mathrm{WHR}_{\mathrm{adj}>50}, \mathrm{PT}=10^{-8}\right.$; $\mathrm{R}=-0.065, P=0.001, \mathrm{Q}=0.023)$. Additional file 4 reports the strong correlations of $\mathrm{WC}_{\text {adj }}$ PRS with these other anthropometric traits. The association between $\mathrm{WC}_{\text {adj }}$ PRS and PTSD appears to be specific to women; no correlation was observed in men (Additional file 5).
Table 2 Top genetic correlations between PTSD and PRS tested

\begin{tabular}{llllll}
\hline PRS & PT & SNP N & $R$ & $P$ value & Q value \\
\hline WC $_{\text {adj }}$ & $10^{-8}$ & 31 & -0.079 & $6.77 \times 10^{-5}$ & 0.011 \\
WHR $_{\text {adj }<50}$ & $10^{-6}$ & 19 & -0.076 & $1.25 \times 10^{-4}$ & 0.011 \\
WHR $_{\text {adj }>50}$ & $10^{-8}$ & 19 & -0.065 & 0.001 & 0.023 \\
AFB & 0.3 & 64,579 & -0.064 & 0.001 & 0.023 \\
WHR & $10^{-7}$ & 32 & -0.064 & 0.001 & 0.023 \\
WHR $_{\text {adj }}$ & $10^{-7}$ & 51 & -0.059 & 0.003 & 0.030 \\
Menarche & $10^{-3}$ & 1510 & 0.049 & 0.013 & 0.074 \\
BMI $_{<50}$ & $10^{-7}$ & 26 & 0.048 & 0.015 & 0.077 \\
HIP & $10^{-5}$ & 94 & 0.045 & 0.024 & 0.108 \\
Height & 0.1 & 25,369 & 0.042 & 0.035 & 0.144 \\
WC & $10^{-8}$ & 20 & 0.034 & 0.082 & 0.266 \\
BMI $_{50}$ & $10^{-5}$ & 104 & 0.033 & 0.099 & 0.306 \\
BMI $_{\text {NEB }}$ & $10^{-4}$ & 316 & 0.032 & 0.103 & 0.312 \\
HIP & $10^{-6}$ & 2 & -0.029 & 0.141 & 0.386 \\
EDU & 0.05 & 13,338 & 0.029 & 0.147 & 0.390 \\
Menopause & $10^{-8}$ & 17 & -0.021 & 0.294 & 0.641 \\
\hline & $10^{-5}$ & 163 & 0.018 & 0.360 & 0.686 \\
\hline
\end{tabular}

Among the significant PRS, the finding related to Age at First Birth (AFB) PRS is the only one that appears to be independent from the $\mathrm{WC}_{\text {adj }}$ signal because its top correlation is due to the cumulative effects of a large number of variants (AFB top-PT $=0.3, \mathrm{SNP} N=64,579$ ) and not to the effect of a small number of strongly associated loci (WC $\mathrm{Wdj}_{\text {tj }}$ top-PT $=10^{-8}, \mathrm{~N}=31$ ). We observed that women with a high genetically predicted AFB have low PTSD risk $(\mathrm{R}=-0.064, P=0.001, \mathrm{Q}=0.023)$. Additional file 6 shows the distribution of AFB PRS in PTSD cases and controls. No association between AFB PRS and PTSD was observed in men (Additional file 7). Since this genetic overlap appear to be related to the additive effect of a large number of variants $(\mathrm{N}=64,579)$, we investigated which molecular mechanisms are shared between PTSD and AFB. The GO enrichment analysis based on AFB results highlighted several potential molecular mechanisms involved $(\mathrm{Q}<0.05$; Additional file 8, respectively). Because we observed a large number of significant $\mathrm{GO}$ enrichments $(\mathrm{Q}<0.05)$ related to the AFB-PTSD result $(\mathrm{N}=40)$, we conducted a similarity-based network analysis among the significant GO enrichments. This analysis highlighted a GO cluster (Additional file 9) related to multiple signaling mechanisms (e.g. calcium-mediated signaling and MAPK cascade).

To investigate further the $\mathrm{WC}_{\mathrm{adj}}$-PTSD genetic correlation, we applied a MR approach to assess the causative mechanisms of this association. We used an inversevariance-weighted method to calculate an overall estimate of the causal effect considering the coefficients related to the association of these variants with PTSD and 
$\mathrm{WC}_{\text {adj }}$ (Fig. 1; Additional file 10). This analysis showed a significant association of effect sizes of $\mathrm{WC}_{\mathrm{adj}}$-associated SNPs with effect sizes estimated in our PTSD sample $(\mathrm{OR}=0.35 ; 95 \%$ confidence interval $[\mathrm{CI}]=0.17-0.73 ; P$ $=0.008)$. This translated to a relative decrease of $64.6 \%$ $(95 \% \mathrm{CI}=27.5-82.7)$ in the risk of PTSD per 1 -SD increase in $\mathrm{WC}_{\mathrm{adj}}$. This result was consistent considering multiple MR approaches (Additional file 11). Furthermore, the consistency between PRS and MR analyses confirms that there is no sample overlap that is biasing our results. Indeed, these two approaches are susceptible to biases from sample overlaps, but the bias would be in opposite directions: risk of false-positive results in PRS and risk of false-negative results in two-sample MR [43]. Considering the MR-Egger regression intercept, we also observe that the markers did not show evidence of pleiotropic effects $\left(\mathrm{P}_{\text {pleiotropy }}=0.979\right)$, suggesting that the association of these variants with PTSD is mediated by their effect on body shape. To investigate further the potential causal relationship between PTSD and female body shape, we tested whether the PTSD PRS correlated with $\mathrm{WC}_{\mathrm{adj}}$. A causal relationship between two traits should show asymmetry in the effect sizes of associated variants [41]. We observed asymmetry between PTSD and $\mathrm{WC}_{\mathrm{adj}}$ : PTSD-associated variants showed no effect on WCadj ( $P>0.10$; Additional file 12) but strongly predicted BMI $\left(P=5.68^{*} 10^{-7}\right)$ and other BMI-related anthropometric traits (Additional file 13). These results support a causal relationship between $\mathrm{WC}_{\mathrm{adj}}$ and PTSD. We tested whether genetically determined female body shape correlated with sexual behaviors in women using female-specific genetic information regarding AFS and NSP (Additional file 10). Considering a penalized robust MR-Egger regression, we observed associations of $\mathrm{WC}_{\mathrm{adj}}$ with AFS (Beta $=-0.25, \mathrm{SE}=0.10, P=0.013$; Fig. 2) and NSP (Beta $=0.16, \mathrm{SE}=0.04, P<0.001$; Fig. 2) with significant evidence of pleiotropy in both results ( $\mathrm{P}_{\text {pleiotropy }}$ $=0.017$ and $<0.001$, respectively), suggesting that the effects of these variants on sexual behaviors could be due to multiple mechanisms.

\section{Discussion}

We found associations of the genetic risk for PTSD in women with genetically determined female body shape and reproductive behaviors. These findings provide novel insight into the mechanisms that may underlie the genetic vulnerability to PTSD in women.

Our strongest result reflects an inverse relationship between genetically determined female body shape and the genetic risk for PTSD. We observed that SNPs associated with anthropometric traits related to body shape independently from BMI (i.e. $\mathrm{WC}_{\text {adj, }}$ WHR, WHR adj, $\mathrm{WHR}_{\text {adj }<50}$,

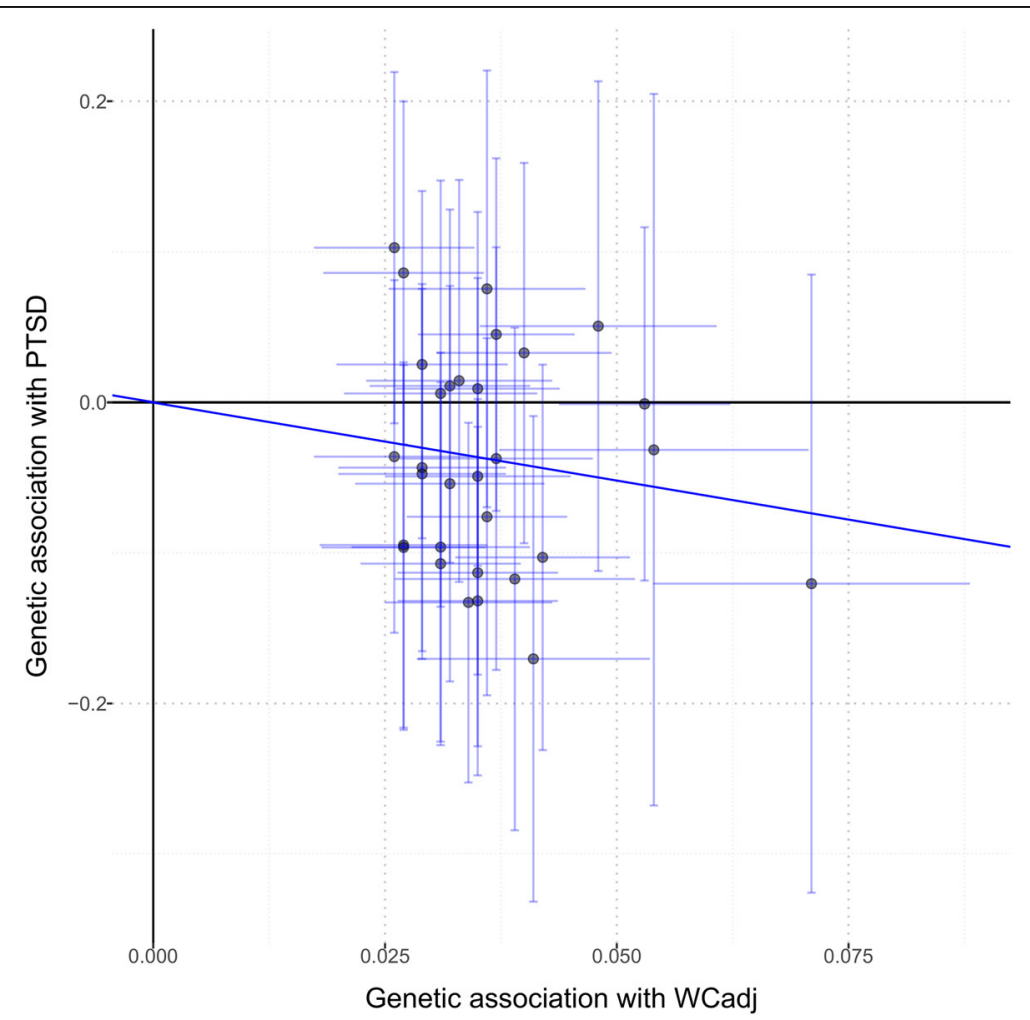

Fig. 1 SNP-exposure (WCadj associations) and SNP-outcome (PTSD associations) coefficients used in the MR analysis. Error bars (95\% Cls) are reported for each association. The solid line represents the inverse-variance-weighted estimate 

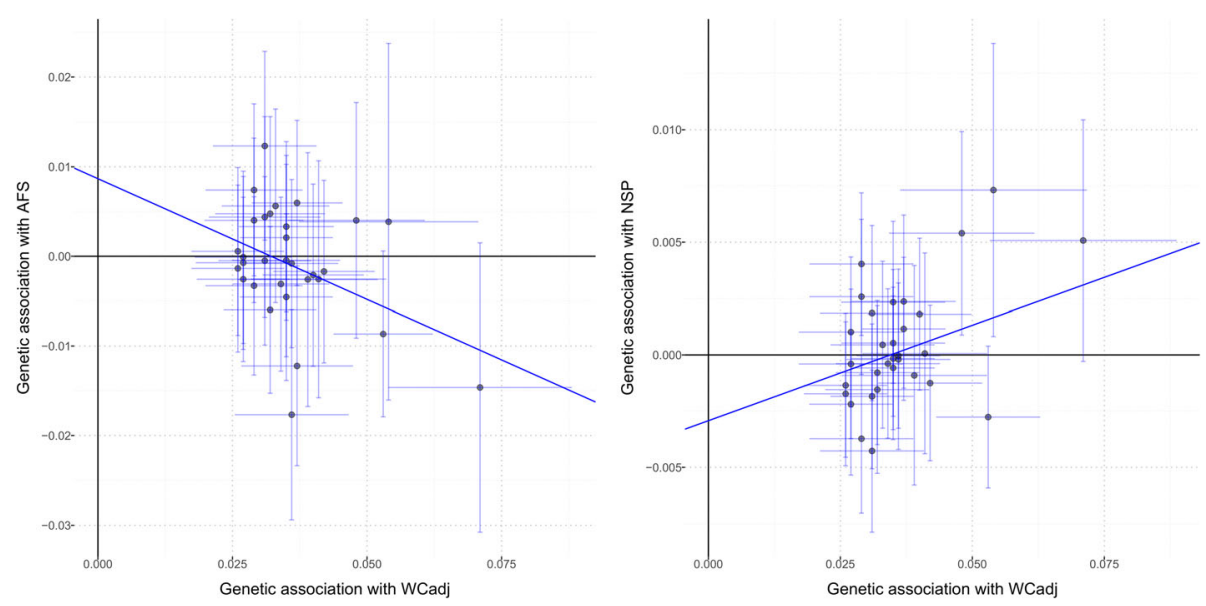

Fig. 2 SNP-exposure (WCadj associations) and SNP-outcome (AFS associations, left; NSP associations, right) coefficients used in the MR analysis. Error bars $(95 \% \mathrm{Cls})$ are reported for each association. The solid line represents the MR-Egger estimate

$\left.W_{H} R_{\text {adj }>50}\right)$ are linked to the risk for PTSD. No analogous association was present in our male cohort. Human body shape is the result of strong evolutionary pressures, including sexual selection, with evident differences in selection between women and men [44]. The dissimilarities between sexes are largely due to adaptation mechanisms related to female reproductive function [44]. To facilitate childbirth, the pelvis is proportionately larger in women than in men. There is also variability in fat accumulation and distribution that are related to women's need for energy to be used during pregnancy and nursing: women have a higher percentage of body fat; men accumulate fat in the abdominal region, while women accumulate fat in hips, buttocks, thighs, and the lower abdomen [45]. Consistent with the presence of different regulatory mechanisms related to human body shape, GWAS from the GIANT consortium identified several loci with sex-specific effects and most of them have larger effects in women than in men [30,32]. Although these sex differences and their regulatory mechanisms are well-known, to our knowledge no large study has investigated the role of genetically determined body shape in women's mental health. In our study, we observed that a genetically determined evolution-related body shape (i.e. low $\mathrm{WC}_{\mathrm{adj}}$, WHR, and $\mathrm{WHR}_{\mathrm{adj}}$ ) was associated with increased risk of PTSD. A small study reported an association between observational (i.e. phenotypically observed) WHR and PTSD concordant with our finding [46]. Our data are most consistent with a causal relationship between $W_{\text {adj }}$ and PTSD in women. Considering MR-Egger regression intercept, we did not observe a pleiotropic effect between $\mathrm{WC}_{\text {adj }}$ and PTSD. Conversely, there was an asymmetry in the effect sizes of associated variants: $\mathrm{WC}_{\mathrm{adj}}$-associated variants affect the risk of PTSD, but PTSD-associated variants do not affect female body shape. Previous evolutionary studies reported that body shape in women is one of the traits related to reproductive attractiveness that has been selected during human evolution [47]. We note that female reproductive attractiveness, which is determined by evolutionary selection pressures, is distinct from socio-cultural norms about female attractiveness that are known to differ over time and place [48]. Our data can be interpreted as supporting that specific anthropometric characteristics may be related to PTSD via their role in increasing the risk of traumatic experiences related to undesired sexual behavior. Further evidence of an apparent causal relationship between $W C_{\text {adj }}$ and experiences related to sexual function is provided by the analysis of AFS and NSP. Genetically determined $\mathrm{WC}_{\text {adj }}$ is associated with AFS and NSP; this provides additional evidence of the relationship between the female body shape and behaviors related to sexual interactions. As indicated by the significant evidence of pleiotropy, the relationship between female body shape and sexual behavior is likely mediated by multiple factors, including behavioral mechanisms and physiological processes related to reproductive function. Unfortunately, we cannot investigate further the relationship between female body shape and traumatic specific experiences, such as sexual assault, because trauma type information is not currently available in the PGC PTSD dataset. We hope to be able to do it in the future. In particular, we believe that longitudinal studies that include detailed life course assessments of traumatic experience will be needed to better understand the role of female body shape in risk of sexual assault or other traumatic event exposure.

Our investigation also identified an association between AFB and PTSD: genetically predicted young maternal AFB is associated with an increase of PTSD risk. This is consistent with epidemiologic observations of an inverse association between maternal AFB and psychiatric distress: mothers giving birth in their teens have an increased risk of a lifetime behavior disorder, PTSD, or an anxiety disorder compared to women who were older 
at first birth [49]. The AFB-PTSD correlation is due to a genome-wide genetic overlap that includes thousands of independent variants, which is different from the body shape result, which was influenced by a small number of variants. This could indicate that these two traits share some pathways involved in their predisposition. Our enrichment analysis identified several molecular processes. Among them, we observed a large GO cluster of signaling mechanisms that includes several that are known to be involved in the response to traumatic experience, such as calcium-mediated signaling and the MAPK cascade $[50,51]$. Observational studies have reported that early trauma exposures are associated with an increased risk of teen pregnancy. In particular, childhood sexual abuse is associated with increased risk for subsequent adolescent pregnancy [52, 53]. In line with this scenario, we note that the AFB GWAS [35] was conducted without excluding birth events from non-consensual sex. For instance, although it accounts for a small fraction of the sample investigated, some of the cohorts included in this previous analysis also included births during childhood. Accordingly, a possible explanation of the AFB-PTSD correlation is that childhood abuse and sexual victimization can contribute to AFB and PTSD, and some of the trauma-response mechanisms involved may be shared among these traits. These mechanisms appear to be different from those related to the $\mathrm{WC}_{\mathrm{adj}}$ results. The relationship between AFB and PTSD seems to be due to a large genetic overlap (i.e. pleiotropy); $\mathrm{WC}_{\mathrm{adj}}$ appears to be involved in a causal mechanism related to women's vulnerability to PTSD (i.e. no pleiotropy).

\section{Conclusions}

In summary, using a genetic approach, we found two associations with the risk of PTSD in women related to body shape and reproductive behaviors. Both mechanisms appear to be female-specific (i.e. there was no such association in men) and they suggest that sex differences in trauma type prevalence and molecular mechanisms of trauma response contribute to the greater vulnerability to PTSD observed in women. Our study demonstrates how genetic research can successfully contribute to the dissection of biological and non-biological mechanisms relevant to women's health.

\section{Additional files}

Additional file 1: Genetic correlations between PTSD and PRS tested in women. (DOCX $19 \mathrm{~kb}$ )

Additional file 2: PTSD genetic correlations tested with LD score regression analysis. (DOCX $12 \mathrm{~kb}$ )

Additional file 3: Distribution of $W_{\text {adj }}$ PRS in PTSD cases and controls. (DOCX $333 \mathrm{~kb})$
Additional file 4: Genetic correlation of WCadj PRS (SNP N $=31, \mathrm{PT}=10^{-8}$ ) with the other anthropometric traits associated with PTSD in women. (DOCX $12 \mathrm{~kb}$ )

Additional file 5: Correlation between $W C_{\text {adj }}$ PRS and PTSD in men. (DOCX $12 \mathrm{~kb}$ )

Additional file 6: Distribution of AFB PRS in PTSD cases and controls. (DOCX $361 \mathrm{~kb})$

Additional file 7: Correlation between AFB PRS and PTSD in men. (DOCX $12 \mathrm{~kb}$ )

Additional file 8: Gene Ontology $(\mathrm{GO})$ enrichments ( $Q$ value $<0.05)$ for variants with AFB GWAS $P<0.3$ and effect direction concordant with the AFB-PTSD correlation. (DOCX $14 \mathrm{~kb}$ )

Additional file 9: Similarity network based on $\mathrm{GO}$ enrichment results related to the AFB-PTSD correlation. Bubble color indicates the GO P value; bubble size indicates the frequency of the GO term in the Gene Ontology Annotation (UniProt-GOA) Database which annotates all UniProt entries with GO terms. Highly similar GO terms are linked by edges in the graph, where the line width indicates the degree of similarity. (DOCX $179 \mathrm{~kb})$

Additional file 10: SNP-exposure and SNP-outcome coefficients of the variants in included in the $W C_{\text {adj }}$ PRS. (DOCX $15 \mathrm{~kb}$ )

Additional file 11: SNP-exposure (WCadj associations) and SNPoutcome (PTSD associations) coefficients used in the MR analysis. The lines (see legend) represents the results observed considering different methods. (DOCX $383 \mathrm{~kb}$ )

Additional file 12: Correlation between PTSD PRS and WC $\mathrm{Cdj}_{\text {. }}$ (DOCX $12 \mathrm{~kb}$ ) Additional file 13: Top results of the reverse PRS analysis of PTSD with respect to the main anthropometric traits. (DOCX $12 \mathrm{~kb}$ )

\section{Abbreviations}

AFB: Age at first birth; AFS: Age at first sexual intercourse; BMl: Body mass index; FDR: False discovery rate; GO: Gene Ontology; GWAS: Genome-wide association study; MR: Mendelian randomization; NSP: Number of sexual partners; PRS: Polygenic risk score; PTSD: Posttraumatic stress disorder; SNP: single nucleotide polymorphism; $W C_{\text {adj: }}$ BMI-adjusted waist circumference; WHR: Waist-hip ratio; WHR adj: WHR adjusted for BMl; $W H R_{\text {adj }<50}$ : WHRadj in women aged $<50$ years; $W H R_{\text {adj }>50: ~} W H R_{\text {adj }}$ in women aged $>50$ years

\section{Acknowledgements}

We thank study participants and research groups contributing to PGC-PTSD for sharing their data. Part of this research has been conducted using the UK Biobank Resource (application reference no. 19664). We acknowledge Naomi Wray of The University of Queensland (Brisbane, Australia) for insightful comments about the manuscript. We thank the members of the cited consortia of genome-wide association studies for making their data available. The members of the Psychiatric Genomics Consortium Posttraumatic Stress Disorder Workgroup are: Laramie E Duncan (Department of Psychiatry, Stanford University, Stanford, CA, USA, Broad Institute of MIT and Harvard, Stanley Center for Psychiatric Research, Boston, MA, USA, The Analytic and Translational Genetics Unit, Massachusetts General Hospital, Boston, MA, USA), Andrew Ratanatharathorn (Department of Epidemiology, Columbia University, New York, NY, USA), Allison E Aiello (Department of Epidemiology, Gillings School of Global Public Health, University of North Carolina, Chapel Hill, Chapel Hill, NC, USA), Lynn M Almli (Department of Psychiatry and Behavioral Sciences, Emory University, Atlanta, GA, USA), Ananda B Amstadter (Department of Psychiatry, Virginia Commonwealth University, Richmond, VA, USA), Allison E Ashley-Koch (Department of Medicine, Duke Molecular Physiology Institute, Duke University Medical Center, Durham, NC, USA), Dewleen G Baker Veterans Affairs San Diego Healthcare System and Veterans Affairs Center of Excellence for Stress and Mental Health, San Diego, CA, USA, Department of Psychiatry, University of California, San Diego, San Diego, CA, USA), Jean C Beckham Veterans Affairs Durham Healthcare System, Durham, NC, USA, Department of Psychiatry and Behavioral Sciences, Duke University Medical Center, Durham, NC, USA), Laura J Bierut (Department of Psychiatry, Washington University School of Medicine, St Louis, MO, USA), Jonathan Bisson (Division of Psychological Medicine and Clinical Neurosciences, Cardiff University, Cardiff, UK), Bekh Bradley (Atlanta VA Medical Center, Atlanta, GA, USA, Department of 
Psychiatry, Emory University, Atlanta, GA, USA), "Chia-Yen Chen" (The Analytic and Translational Genetics Unit, Massachusetts General Hospital, Boston, MA, USA Psychiatric and Neurodevelopmental Genetics Unit, Center for Human, enetic Research, and Department of Psychiatry, Massachusetts General Hospital, Boston, MA, USA, Department of Psychiatry, Harvard University, Cambridge, MA, USA), Shareefa Dalvie (Division of Human Genetics, University of Cape Town, Cape Town, South Africa), Lindsay A Farrer (Biomedical Genetics, Boston University School of Medicine, Boston, MA, USA), Sandro Galea (Boston University School of Public Health, Boston, MA, USA), Melanie E Garrett (Department of Medicine, Duke Molecular Physiology Institute, Duke University Medical Center, Durham, NC, USA), Joel E Gelernter (Department of Psychiatry, Yale University School of Medicine and VA CT Healthcare System, New Haven, CT, USA), Guia Guffanti (Department of Psychiatry, Harvard University, Cambridge, MA, USA, Department of Psychiatry, McLean Hospital, Belmont, MA, USA), Michael A Hauser (Department of Medicine, Duke Molecular Physiology Institute, Duke University Medical Center, Durham, NC, USA), Eric O Johnson (RTI International, Research Triangle Park, NC, USA), Ronald C Kessler (Department of Health Care Policy, Harvard Medical School, Boston, MA, USA), Nathan A Kimbrel (Veterans Affairs Durham Healthcare System, Durham, NC, USA, Department of Psychiatry and Behavioral Sciences, Duke University Medical Center, Durham, NC, USA), Anthony King (Department of Psychiatry, University of Michigan, Ann Arbor, MI, USA), Nastassja Koen (Department of Psychiatry and Mental Health, University of Cape Town, Cape Town, South Africa, MRC Unit on Anxiety \& Stress Disorders, Groote Schuur Hospital, Cape Town, South Africa), Henry R Kranzler (Department of Psychiatry, University of Pennsylvania Perelman School of Medicine and VISN 4 MIRECC, Crescenz VAMC, Philadelphia, PA, USA), Mark W Logue (VA Boston Healthcare System, Jamaica Plain, MA, USA, Department of Medicine, Boston University School of Medicine, Boston, MA, USA), Adam X Maihofer (Department of Psychiatry, University of California, San Diego, La Jolla, CA, USA), Alicia R Martin (Broad Institute of MIT and Harvard, Stanley Center for Psychiatric Research, Boston, MA, USA, The Analytic and Translational Genetics Unit, Massachusetts General Hospital, Boston, MA, USA), Mark W Miller (VA Boston Healthcare System, Jamaica Plain, MA, USA, Department of Psychiatry, Boston University School of Medicine, Boston, MA, USA), Rajendra A Morey (Department of Psychiatry and Behavioral Sciences, Duke University Medical Center, Durham, NC, USA, Durham VA Medical Center, Durham, NC, USA), Nicole R Nugent (Division of Behavioral Genetics, Department of Psychiatry, Rhode Island Hospital, Providence, Rl, USA, Department of Psychiatry and Human Behavior, Alpert Medical School of Brown University, Providence, RI, USA), John P Rice (Department of Psychiatry, Washington University, St Louis, MO, USA), Stephan Ripke (Broad Institute of MIT and Harvard, Stanley Center for Psychiatric Research, Boston, MA, USA, The Analytic and Translational Genetics Unit, Massachusetts General Hospital, Boston, MA, USA, Department of Psychiatry and Psychotherapy, Charité, Campus Mitte, Berlin, Germany), Andrea L Roberts (Department of Social and Behavioral Sciences, Harvard T. H. Chan School of Public Health Cambridge, MA, USA), Nancy L Saccone (Department of Genetics, Washington University, St Louis, MO, USA), Jordan W Smoller (Broad Institute of MIT and Harvard, Stanley Center for Psychiatric Research, Boston, MA, USA, Psychiatric and Neurodevelopmental Genetics Unit, Center for Human Genetic, Research, and Department of Psychiatry, Massachusetts General Hospital, Boston, MA, USA), Daniel J Stein (Department of Psychiatry and Mental Health, University of Cape Town, Cape Town, South Africa, MRC Unit on Anxiety \& Stress Disorders, Groote Schuur Hospital, Cape Town, South Africa), Murray B Stein (Department of Psychiatry, University of California, San Diego, La Jolla, CA, USA, Veterans Affairs San Diego Healthcare System, San Diego, CA, USA, Department of Family Medicine and Public Health, University of California, San Diego, La Jolla, CA, USA), Jennifer A Sumner (Center for Cardiovascular Behavioral Health, Columbia University Medical Center, New York, NY, USA), Monica Uddin (Department of Psychology and Carl R. Woese Institute for Genomic Biology, University of Illinois at Urbana-Champaign, Urbana, IL, USA), Robert J Ursano (Center for the Study of Traumatic Stress, Department of Psychiatry, Uniformed Services University of the Health Sciences, Bethesda, MD, USA), Derek E Wildman (Department of Molecular \& Integrative Physiology and Carl R. Woese Institute for Genomic Biology, University of Illinois at Urbana-Champaign, Urbana, IL, USA), Rachel Yehuda (James J. Peters Bronx Veterans Affairs and Department of Psychiatry, Icahn School of Medicine at Mount Sinai, Bronx, NY, USA, Department of Neuroscience, Icahn School of Medicine at Mount Sinai, Bronx, NY, USA), Hongyu Zhao (Department of Biostatistics, Yale University, New Haven, CT, USA), Mark J Daly (Broad Institute of MIT and Harvard, Stanley Center for Psychiatric Research, Boston, MA, USA, The Analytic and Translational Genetics Unit, Massachusetts General Hospital, Boston, MA, USA), Israel Liberzon (Department of Psychiatry, University of Michigan, Ann Arbor, Ml, USA, VA Ann Arbor Health System, Ann Arbor, MI, USA), Kerry J Ressler (Department of Psychiatry, Harvard University, Cambridge, MA, USA,
Department of Psychiatry, McLean Hospital, Belmont, MA, USA), Caroline M Nievergelt (Veterans Affairs San Diego Healthcare System and Veterans Affairs Center of Excellence for Stress and Mental Health, San Diego, CA, USA, Department of Psychiatry, University of California, San Diego, San Diego, CA, USA), Karestan C Koenen (Broad Institute of MIT and Harvard, Stanley Center for Psychiatric Research, Boston, MA, USA, Psychiatric and Neurodevelopmental Genetics Unit, Center for Human Genetic Research, and Department of Psychiatry, Massachusetts General Hospital, Boston, MA, USA, Department of Epidemiology, Harvard T. H. Chan School of Public Health, Cambridge, MA, USA).

\section{Funding}

This study was supported by National Institutes of Health grants R01 MH106595 and U01 MH109532, and the VA National Center for PTSD Research.

\section{Availability of data and materials}

All data generated during this study are included in this published article and its Additional files.

\section{Authors' contributions}

$R P, M B S$, and JG were involved in study design. $A B A, L M A, D G B, L J B, B B$, LAF, EOJ, AK, HRK, AXM, JPR, ALR, NLS, HZ, IL, KJR, CMN, KCK, and JG were involved in cohort ascertainment, phenotypic characterization, genotyping, and/or recruitment. RP carried out statistical analysis. RP wrote the first draft of the manuscript and all authors contributed to the final version of the manuscript. All authors read and approved the final manuscript.

\section{Ethics approval and consent to participate}

This study is based on the PGC-PTSD data freeze 1, which is an anonymized dataset. The cohorts contributing to the PGC-PTSD workgroup included in the present analysis are: Collaborative Genetic Study of Nicotine Dependence; Family Study of Cocaine Dependence; Yale-Penn; Grady Trauma Project; Marine Resilience Study; Nurses' Health Study; and Ohio National Guard. Each of the contributing studies has an institutional review board-approved protocol to investigate the genetics of PTSD and provided the data consistent with their approved protocols. All studies involved in the PGC-PTSD workgroup comply with the Declaration of Helsinki. Additionally, we used GWAS summary statistics from the GIANT, Reprogen, and SSGAC consortia, which shared this information in accordance with the protocols approved for the cohorts participating in these studies.

\section{Consent for publication}

Not applicable.

\section{Competing interests}

Dr. Stein has in the last three years been a consultant for Actelion Pharmaceuticals, Healthcare Management Technologies, Janssen, Pfizer, Resilience Therapeutics, Tonix Pharmaceuticals, and Oxeia Biopharmaceuticals. Dr. Kranzler has been an advisory board member, consultant, or CME speaker for Indivior and Lundbeck. He is also a member of the American Society of Clinical Psychopharmacology's Alcohol Clinical Trials Initiative, which was supported in the last three years by AbbVie, Alkermes, Ethypharm, Indivior, Lilly, Lundbeck, Pfizer, Arbor, and Amygdala Neurosciences. Dr. Bierut is listed as an inventor on Issued US Patent $8,080,371$, "Markers for Addiction" covering the use of certain SNPs in determining the diagnosis, prognosis, and treatment of addiction. Dr Liberzon has been a consultant for ARMGO Pharmaceutical, Sunovion Pharmaceutical, and Trimaran Pharma. The remaining authors declare that they have no competing interests.

\section{Publisher's Note}

Springer Nature remains neutral with regard to jurisdictional claims in published maps and institutional affiliations.

\section{Author details}

${ }^{1}$ Department of Psychiatry, Yale University School of Medicine and VA CT Healthcare Center, 116A2, 950 Campbell Avenue, West Haven, CT 06516, USA. ²Department of Psychiatry, Virginia Commonwealth University, Richmond, VA, USA. ${ }^{3}$ Department of Psychiatry, University of California San Diego, La Jolla, CA, USA. ${ }^{4}$ Department of Family Medicine and Public Health, 
University of California San Diego, La Jolla, CA, USA. ${ }^{5}$ Veterans Affairs San Diego Healthcare System and Veterans Affairs Center of Excellence for Stress and Mental Health, La Jolla, CA, USA. ${ }^{6}$ Department of Psychiatry and Behavioral Sciences, Emory University, Atlanta, GA, USA. ${ }^{7}$ Department of Psychiatry, Washington University School of Medicine, St. Louis, MO, USA. ${ }^{8}$ Atlanta VA Medical Center, Atlanta, GA, USA. ${ }^{9}$ Department of Medicine, Biomedical Genetics Division, Boston University School of Medicine, Boston MA, USA. ${ }^{10}$ Fellow Program and Behavioral Health and Criminal Justice Division RTI International, Research Triangle Park, NC, USA. ${ }^{11}$ Department of Psychiatry, University of Michigan, Ann Arbor, MI, USA. ${ }^{12}$ Department of Psychiatry, University of Pennsylvania Perelman School of Medicine and VISN 4 MIRECC, Crescenz VAMC, Philadelphia, PA, USA. ${ }^{13}$ Department of Environmental Health, Harvard T. H. Chan School of Public Health, Boston, MA, USA. ${ }^{14}$ Department of Genetics, Washington University School of Medicine, St. Louis, MO, USA. ${ }^{15}$ Department of Biostatistics, Yale University, New Haven, CT, USA. ${ }^{16}$ VA Ann Arbor Health System, Ann Arbor, MI, USA. ${ }^{17}$ Department of Psychiatry, Harvard University, Cambridge, MA, USA. ${ }^{18}$ Department of Psychiatry, McLean Hospital, Belmont, MA, USA. ${ }^{19}$ Department of Epidemiology, Harvard TH Chan School of Public Health, Boston, MA, USA. ${ }^{20}$ Psychiatric and Neurodevelopmental Genetics Unit, Center for Human Genetic Research, and Department of Psychiatry, Massachusetts General Hospital, Boston, MA, USA. ${ }^{21}$ Broad Institute of MIT and Harvard, Stanley Center for Psychiatric Research, Boston, MA, USA. ${ }^{22}$ Departments of Neuroscience and of Genetics, Yale University School of Medicine, New Haven, CT, USA.

Received: 28 July 2017 Accepted: 6 November 2017

Published online: 27 November 2017

\section{References}

1. Pitman RK, Rasmusson AM, Koenen KC, Shin LM, Orr SP, Gilbertson MW, et al. Biological studies of post-traumatic stress disorder. Nat Rev Neurosci. 2012;13:769-87.

2. American Psychiatric Association. Trauma- and stressor-related disorders. In: Diagnostic and Statistical Manual of Mental Disorder. Washington, DC: American Psychiatric Association; 2013.

3. Kessler RC, Sonnega A, Bromet E, Hughes M, Nelson CB. Posttraumatic stress disorder in the National Comorbidity Survey. Arch Gen Psychiatry. 1995;52: 1048-60.

4. Bangasser DA, Valentino RJ. Sex differences in molecular and cellular substrates of stress. Cell Mol Neurobiol. 2012;32:709-23.

5. Olff M, Langeland W, Draijer N, Gersons BP. Gender differences in posttraumatic stress disorder. Psychol Bull. 2007;133:183-204.

6. Kilpatrick DG, Resnick HS, Milanak ME, Miller MW, Keyes KM, Friedman MJ. National estimates of exposure to traumatic events and PTSD prevalence using DSM-IV and DSM-5 criteria. J Trauma Stress. 2013;26:537-47.

7. Cortina LM, Kubiak SP. Gender and posttraumatic stress: sexual violence as an explanation for women's increased risk. J Abnorm Psychol. 2006; 115:753-9.

8. Sartor CE, McCutcheon W, Pommer NE, Nelson EC, Grant JD, Duncan AE, et al. Common genetic and environmental contributions to post-traumatic stress disorder and alcohol dependence in young women. Psychol Med. 2011:41:1497-505.

9. True WR, Rice J, Eisen SA, Heath AC, Goldberg J, Lyons MJ, et al. A twin study of genetic and environmental contributions to liability for posttraumatic stress symptoms. Arch Gen Psychiatry. 1993;50:257-64.

10. Duncan LE, Ratanatharathorn A, Aiello AE, Almli LM, Amstadter AB, AshleyKoch $A E$, et al. Largest GWAS of PTSD ( $N=20$ 070) yields genetic overlap with schizophrenia and sex differences in heritability. Mol Psychiatry. 2017. http://doi.org/10.1038/mp.2017.77.

11. Li SH, Graham BM. Why are women so vulnerable to anxiety, trauma-related and stress-related disorders? The potential role of sex hormones. Lancet Psychiatry. 2017;4:73-82.

12. Maddox SA, Kilaru V, Shin J, Jovanovic T, Almli LM, Dias BG, et al. Estrogendependent association of HDAC4 with fear in female mice and women with PTSD. Mol Psychiatry. 2017. http://doi.org/10.1038/mp.2016.250.

13. Pasaniuc B, Price AL. Dissecting the genetics of complex traits using summary association statistics. Nat Rev Genet. 2017:18:117-27.

14. Polimanti R, Gelernter J, Stein DJ. Genetically determined schizophrenia is not associated with impaired glucose homeostasis. Schizophr Res. 2017. http://doi.org/10.1016/j.schres.2017.10.033.
15. Kubzansky LD, Bordelois P, Jun HJ, Roberts AL, Cerda M, Bluestone N, et al. The weight of traumatic stress: a prospective study of posttraumatic stress disorder symptoms and weight status in women. JAMA Psychiat. 2014;71:44-51.

16. Yonkers KA, Smith MV, Forray A, Epperson CN, Costello D, Lin H, et al. Pregnant women with posttraumatic stress disorder and risk of preterm birth. JAMA Psychiat. 2014;71:897-904.

17. Boyraz G, Granda R, Baker CN, Tidwell LL, Waits JB. Posttraumatic stress, effort regulation, and academic outcomes among college students: A longitudinal study. J Couns Psychol. 2016;63:475-86.

18. Bulik-Sullivan B, Finucane HK, Anttila V, Gusev A, Day FR, Loh PR, et al. An atlas of genetic correlations across human diseases and traits. Nat Genet. 2015;47:1236-41.

19. Bierut LJ, Strickland JR, Thompson JR, Afful SE, Cottler LB. Drug use and dependence in cocaine dependent subjects, community-based individuals, and their siblings. Drug Alcohol Depend. 2008;95:14-22.

20. Bierut LJ, Madden PA, Breslau N, Johnson EO, Hatsukami D, Pomerleau OF, et al. Novel genes identified in a high-density genome wide association study for nicotine dependence. Hum Mol Genet. 2007;16:24-35.

21. Xie P, Kranzler HR, Yang C, Zhao H, Farrer LA, Gelernter J. Genome-wide association study identifies new susceptibility loci for posttraumatic stress disorder. Biol Psychiatry. 2013;74:656-63.

22. Almli LM, Stevens JS, Smith AK, Kilaru V, Meng Q, Flory J, et al. A genomewide identified risk variant for PTSD is a methylation quantitative trait locus and confers decreased cortical activation to fearful faces. Am J Med Genet B Neuropsychiatr Genet. 2015;168B:327-36.

23. Nievergelt CM, Maihofer AX, Mustapic M, Yurgil KA, Schork NJ, Miller MW, et al. Genomic predictors of combat stress vulnerability and resilience in U.S. Marines: A genome-wide association study across multiple ancestries implicates PRTFDC1 as a potential PTSD gene. Psychoneuroendocrinology. 2015;51:459-71.

24. Koenen KC, De Vivo I, Rich-Edwards J, Smoller JW, Wright RJ, Purcell SM. Protocol for investigating genetic determinants of posttraumatic stress disorder in women from the Nurses' Health Study II. BMC Psychiatry. 2009;9:29.

25. Liberzon I, King AP, Ressler KJ, Almli LM, Zhang P, Ma ST, et al. Interaction of the ADRB2 gene polymorphism with childhood trauma in predicting adult symptoms of posttraumatic stress disorder. JAMA Psychiat. 2014;71:1174-82.

26. Chang CC, Chow CC, Tellier LC, Vattikuti S, Purcell SM, Lee JJ. Secondgeneration PLINK: rising to the challenge of larger and richer datasets. Gigascience. 2015;4:7.

27. Euesden J, Lewis CM, O'Reilly PF. PRSice: Polygenic Risk Score software. Bioinformatics. 2015;31:1466-8.

28. International Schizophrenia Consortium, Purcell SM, Wray NR, Stone JL, Visscher PM, O'Donovan MC, et al. Common polygenic variation contributes to risk of schizophrenia and bipolar disorder. Nature. 2009:460:748-52.

29. Locke AE, Kahali B, Berndt SI, Justice AE, Pers TH, Day FR, et al. Genetic studies of body mass index yield new insights for obesity biology. Nature. 2015:518:197-206.

30. Winkler TW, Justice AE, Graff M, Barata L, Feitosa MF, Chu S, et al. The influence of age and sex on genetic associations with adult body size and shape: a large-scale genome-wide interaction study. PLoS Genet. 2015;11:e1005378.

31. Wood AR, Esko T, Yang J, Vedantam S, Pers TH, Gustafsson S, et al. Defining the role of common variation in the genomic and biological architecture of adult human height. Nat Genet. 2014;46:1173-86.

32. Shungin D, Winkler TW, Croteau-Chonka DC, Ferreira T, Locke AE, Magi R, et al. New genetic loci link adipose and insulin biology to body fat distribution. Nature. 2015;518:187-96.

33. Perry JR, Day F, Elks CE, Sulem P, Thompson DJ, Ferreira T, et al. Parent-oforigin-specific allelic associations among 106 genomic loci for age at menarche. Nature. 2014;514:92-7.

34. Day FR, Ruth KS, Thompson DJ, Lunetta KL, Pervjakova N, Chasman DI, et al. Large-scale genomic analyses link reproductive aging to hypothalamic signaling, breast cancer susceptibility and BRCA1-mediated DNA repair. Nat Genet. 2015:47:1294-303

35. Barban N, Jansen R, de Vlaming R, Vaez A, Mandemakers JJ, Tropf FC, et al. Genome-wide analysis identifies 12 loci influencing human reproductive behavior. Nat Genet. 2016;48:1462-72

36. Okbay A, Beauchamp JP, Fontana MA, Lee JJ, Pers TH, Rietveld CA, et al. Genome-wide association study identifies 74 loci associated with educational attainment. Nature. 2016:533:539-42.

37. Mi H, Huang X, Muruganujan A, Tang H, Mills C, Kang D, et al. PANTHER version 11: expanded annotation data from Gene Ontology and Reactome pathways, and data analysis tool enhancements. Nucleic Acids Res. 2017;45:D183-D9. 
38. Supek F, Bosnjak M, Skunca N, Smuc T. REVIGO summarizes and visualizes long lists of gene ontology terms. PLoS One. 2011;6:e21800.

39. Burgess S, Scott RA, Timpson NJ, Davey Smith G, Thompson SG, EPIC-InterAct Consortium. Using published data in Mendelian randomization: a blueprint for efficient identification of causal risk factors. Eur J Epidemiol. 2015;30:543-52.

40. Bowden J, Davey Smith G, Burgess S. Mendelian randomization with invalid instruments: effect estimation and bias detection through Egger regression. Int J Epidemiol. 2015;44:512-25.

41. Pickrell JK, Berisa T, Liu JZ, Segurel L, Tung JY, Hinds DA. Detection and interpretation of shared genetic influences on 42 human traits. Nat Genet. 2016:48:709-17.

42. Day FR, Helgason H, Chasman DI, Rose LM, Loh PR, Scott RA, et al. Physical and neurobehavioral determinants of reproductive onset and success. Nat Genet. 2016;48:617-23.

43. Burgess S, Davies NM, Thompson SG. Bias due to participant overlap in twosample Mendelian randomization. Genet Epidemiol. 2016;40:597-608.

44. Polimanti $\mathrm{R}$, Yang BZ, Zhao H, Gelernter J. Evidence of polygenic adaptation in the systems genetics of anthropometric traits. PLoS One. 2016;11:e0160654

45. Fuente-Martin E, Argente-Arizon P, Ros P, Argente J, Chowen JA. Sex differences in adipose tissue: It is not only a question of quantity and distribution. Adipocyte. 2013:2:128-34.

46. Gandubert C, Scali J, Ancelin ML, Carriere I, Dupuy AM, Bagnolini G, et al. Biological and psychological predictors of posttraumatic stress disorder onset and chronicity. A one-year prospective study. Neurobiol Stress. 2016;3:61-7.

47. Jasienska G, Ziomkiewicz A, Ellison PT, Lipson SF, Thune I. Large breasts and narrow waists indicate high reproductive potential in women. Proc Biol Sci. 2004;271:1213-7.

48. Strahan EJ, Wilson AE, Cressman KE, Buote VM. Comparing to perfection: How cultural norms for appearance affect social comparisons and selfimage. Body Image. 2006;3:211-27.

49. Tabet M, Flick LH, Cook CA, Xian H, Chang JJ. Age at first birth and psychiatric disorders in low-income pregnant women. J Womens Health (Larchmt). 2016;25:810-7.

50. Hauger RL, Olivares-Reyes JA, Dautzenberg FM, Lohr JB, Braun S, Oakley RH. Molecular and cell signaling targets for PTSD pathophysiology and pharmacotherapy. Neuropharmacology. 2012;62:705-14.

51. Singewald N, Schmuckermair C, Whittle N, Holmes A, Ressler KJ. Pharmacology of cognitive enhancers for exposure-based therapy of fear, anxiety and trauma-related disorders. Pharmacol Ther. 2015;149:150-90.

52. Noll JG, Shenk CE, Putnam KT. Childhood sexual abuse and adolescent pregnancy: a meta-analytic update. J Pediatr Psychol. 2009;34:366-78.

53. Boyer D, Fine D. Sexual abuse as a factor in adolescent pregnancy and child maltreatment. Fam Plann Perspect. 1992;24:4-11. 9.

\section{Submit your next manuscript to BioMed Central and we will help you at every step:}

- We accept pre-submission inquiries

- Our selector tool helps you to find the most relevant journal

- We provide round the clock customer support

- Convenient online submission

- Thorough peer review

- Inclusion in PubMed and all major indexing services

- Maximum visibility for your research

Submit your manuscript at www.biomedcentral.com/submit 